\title{
E/B decomposition of CMB polarization pattern of incomplete sky: a pixel space approach
}

\author{
J. Kim and P. Naselsky \\ Niels Bohr Institute \& Discovery Center, Blegdamsvej 17, 2100 Copenhagen, Denmark \\ e-mail: jkim@nbi.dk \\ Received 6 April 2010 / Accepted 26 June 2010

\begin{abstract}
CMB polarization pattern may be decomposed into gradient-like (E) and curl-like (B) mode, which provide invaluable information respectively. We have investigated $\mathrm{E} / \mathrm{B}$ decomposition in pixel space. We find $\mathrm{E} / \mathrm{B}$ mixing due to incomplete sky is localized in pixel-space, and negligible in the regions far away from the masked area. By estimating the expected local leakage power, we have diagnosed ambiguous pixels. Our criteria for ambiguous pixels (i.e. $r_{\mathrm{c}}$ ) is associated with the tensor-to-scalar ratio of B mode power spectrum, which the leakage power is comparable to. By setting $r_{\mathrm{c}}$ to a lower value, we may reduce leakage level, but reduce sky fraction at the same time. Therefore, we have solved $\partial \Delta C_{l} / \partial r_{\mathrm{c}}=0$, and obtained the optimal $r_{\mathrm{c}}$, which minimizes the estimation uncertainty, given a foreground mask and noise level. We have applied our method to a simulated map blocked by a foreground (diffuse + point source) mask. Our simulation shows leakage power is smaller than primordial (i.e. unlensed) B mode power spectrum of tensor-to-scalar ratio $r \sim 1 \times 10^{-3}$ at wide range of multipoles $(50 \lesssim l \lesssim 2000$ ), while allowing us to retain sky fraction $\sim 0.48$.
\end{abstract}

Key words. methods: data analysis - cosmic background radiation

\section{Introduction}

Over the past years, CMB polarization has been measured by several experiments and is being measured by the Planck surveyor (Kovac et al. 2002; Leitch et al. 2002; Pryke et al. 2002a,b Halverson et al. 2002; Leitch et al. 2005; Ade \& et al. 2008; Pryke \& et al. 2009; Hinderks et al. 2009; Brown \& et al. 2009; The Planck Collaboration 2006). CMB polarization pattern may be considered as the sum of gradient-like E mode and curllike B mode (Zaldarriaga \& Seljak 1997; Kamionkowski et al. 1997). In the standard model, B mode polarization is not produced by scalar perturbation, but solely by tensor perturbation. Therefore, measurement of $\mathrm{B}$ mode polarization makes it possible to probe the universe on the energy scale at inflationary period (Kamionkowski et al. 1997; Zaldarriaga \& Seljak 1997; Dodelson 2003; Liddle \& Lyth 2000; Mukhanov 2005). In most inflationary models, tensor-to-scalar ratio $r$ is much smaller than one, and the WMAP 7 year data imposes an upper bound on $r<0.36$ at $95 \%$ confidence level (Larson et al. 2010; Komatsu et al. 2010).

Besides instrument noise, there are complications, which limits detectability of tensor perturbation. Imperfection in removing foreground and gravitational lensing imposes observational limit on tensor-scalar-ratio: $r \sim 10^{-4}$ and $r \sim 3 \times 10^{-5}$ respectively (Seljak \& Hirata 2004; Tucci et al. 2005). Due to the nature of the observation or heavy foreground contamination, reliable of estimation on CMB polarization signal is not available over a whole sky. Incomplete sky coverage leads to E/B mixing, and very significantly limit our capacity to measure tensor perturbation as well (Bunn et al. 2003). Therefore, there have been various efforts to understand and reduce E/B mixing (Kim 2007a,b; Bunn et al. 2003; Lewis et al. 2002; Lewis 2003; Smith 2006).
It is best to implement $\mathrm{E} / \mathrm{B}$ decomposition in map space, since diffuse foregrounds and point sources are well-localized in map space, and their spatial information are known relatively better than other properties. In this paper, we investigate E/B decomposition in pixel space. Our investigation shows that $\mathrm{E} / \mathrm{B}$ mixing is highly localized in pixel space. Therefore, we may reduce $\mathrm{E} / \mathrm{B}$ mixing effectively by excluding the ambiguous pixels. We have applied our method to simulated maps partially blocked by a foreground (diffuse + point source) mask. After excluding ambiguous pixels, we find that leakage power in retained pixels (sky fraction $\sim 0.48$ ) is smaller than primordial (i.e. unlensed) B mode power spectrum of tensor-to-scalar ratio $r \sim 1 \times 10^{-3}$ at wide range of multipoles $(50 \lesssim l \lesssim 2000)$.

The outline of this paper is as follows. In Sect. 2, we discuss all-sky analysis of CMB polarization. In Sect. 3, we derive $\mathrm{E} / \mathrm{B}$ decomposition in pixel space. In Sect. 4, we discuss the application to cut sky, and the method to diagnose ambiguous pixels. In Sects. 5 and 6, we present our simulation result. In Sect. 7 , we summarize our investigation. In Appendix A, we discuss error analysis of pseudo $C_{l}$ estimation, and show interpixel noise correlation may be neglected.

\section{Stokes parameters}

The state of polarization is described by Stokes parameter (Kraus 1986; Rohlfs \& Wilson 2003). Since Thompson scattering does not generate circular polarization, Stokes parameter $\mathrm{Q}$ and $\mathrm{U}$ are sufficient to describe $\mathrm{CMB}$ polarization (Dodelson 2003). Stokes parameter $Q$ and $U$ transform under rotation of an angle $\psi$ on the plane perpendicular to direction $\hat{\boldsymbol{n}}$ (Zaldarriaga \& Seljak 1997; Zaldarriaga 1998):

$(Q \pm \mathrm{i} U)^{\prime}(\hat{\boldsymbol{n}})=\mathrm{e}^{\mp 2 \mathrm{i} \psi}(Q \pm \mathrm{i} U)(\hat{\boldsymbol{n}})$. 
Therefore, all-sky Stokes parameters may be decomposed into spin \pm 2 spherical harmonics (Zaldarriaga \& Seljak 1997) as follows:

$Q(\hat{\boldsymbol{n}}) \pm \mathrm{i} U(\hat{\boldsymbol{n}})=\sum_{l, m} a_{ \pm 2, l m \pm 2} Y_{l m}(\hat{\boldsymbol{n}})$,

where the decomposition coefficients $a_{ \pm 2, l m}$ are obtained by:

$a_{ \pm 2, l m}=\int[Q(\hat{\boldsymbol{n}}) \pm \mathrm{i} U(\hat{\boldsymbol{n}})]_{ \pm 2} Y_{l m}^{*}(\hat{\boldsymbol{n}}) \mathrm{d} \hat{\boldsymbol{n}}$.

Though the quantity shown in Eq. (2) has direct association with physical observables (i.e. Stokes parameters), rotational variance leads to computational complication. Therefore, two real scalar quantities, termed "E" and "B" mode, are often built out of $Q(\hat{\boldsymbol{n}}) \pm \mathrm{i} U(\hat{\boldsymbol{n}})$ (Kamionkowski et al. 1997; Zaldarriaga \& Seljak 1997):

$$
\begin{aligned}
E(\hat{\boldsymbol{n}}) & =-\frac{1}{2}\left[\bar{\partial}^{2}(Q(\hat{\boldsymbol{n}})+\mathrm{i} U(\hat{\boldsymbol{n}}))+\partial^{2}(Q(\hat{\boldsymbol{n}})-\mathrm{i} U(\hat{\boldsymbol{n}}))\right], \\
& =\sum_{l m} \sqrt{\frac{(l+2) !}{(l-2) !}} a_{\mathrm{E}, l m} Y_{l m}(\hat{\boldsymbol{n}}), \\
B(\hat{\boldsymbol{n}}) & =\frac{\mathrm{i}}{2}\left[\bar{\partial}^{2}(Q(\hat{\boldsymbol{n}})+\mathrm{i} U(\hat{\boldsymbol{n}}))-\partial^{2}(Q(\hat{\boldsymbol{n}})-\mathrm{i} U(\hat{\boldsymbol{n}}))\right], \\
& =\sum_{l m} \sqrt{\frac{(l+2) !}{(l-2) !}} a_{\mathrm{B}, l m} Y_{l m}(\hat{\boldsymbol{n}}),
\end{aligned}
$$

where $\bar{\partial}$ and $\delta$ refer to lowering and raising operator respectively (Zaldarriaga \& Seljak 1997). The explicit expression of $\bar{\partial}$ and $\mathscr{\partial}$ are given as follows (Zaldarriaga \& Seljak 1997):

$$
\begin{aligned}
& \partial_{s} f(\theta, \phi)=-\sin ^{s} \theta\left[\frac{\partial}{\partial \theta}+\mathrm{i} \csc \theta \frac{\partial}{\partial \phi}\right] \sin ^{-s} \theta_{s} f(\theta, \phi), \\
& \bar{\partial}_{s} f(\theta, \phi)=-\sin ^{-s} \theta\left[\frac{\partial}{\partial \theta}-\mathrm{i} \csc \theta \frac{\partial}{\partial \phi}\right] \sin ^{s} \theta_{s} f(\theta, \phi),
\end{aligned}
$$

where ${ }_{s} f(\theta, \phi)$ is an arbitrary spin $s$ function. The decomposition coefficients of $\mathrm{E}$ and $\mathrm{B}$ mode are related to $a_{ \pm 2, l m}$ (Zaldarriaga \& Seljak 1997) as follows:

$a_{\mathrm{E}, l m}=-\left(a_{2, l m}+a_{-2, l m}\right) / 2$,

$a_{\mathrm{B}, l m}=\mathrm{i}\left(a_{2, l m}-a_{-2, l m}\right) / 2$.

For a Gaussian seed fluctuation model, decomposition coefficients of E and B mode satisfy the following statistical properties:

$$
\begin{aligned}
\left\langle a_{\mathrm{E}, l m}^{*} a_{\mathrm{E}, l^{\prime} m^{\prime}}\right\rangle & =C_{l}^{\mathrm{EE}} \delta_{l l^{\prime}} \delta_{m m^{\prime}}, \\
\left\langle a_{\mathrm{B}, l m}^{*} a_{\mathrm{B}, l^{\prime} m^{\prime}}\right\rangle & =C_{l}^{\mathrm{BB}} \delta_{l l^{\prime}} \delta_{m m^{\prime}},
\end{aligned}
$$

where $\langle\ldots\rangle$ denotes an ensemble average. In Fig. 1, we show unlensed $C_{l}^{\mathrm{EE}}$ and $C_{l}^{\mathrm{BB}}$ of the WMAP concordance $\Lambda \mathrm{CDM}$ model for various tensor-to-scalar ratio $r$.

\section{E/B decomposition in pixel space}

In this section, we are going to derive a pixel-space analogue of E/B decomposition. Using Eqs. (3), (6) and (7), we may easily
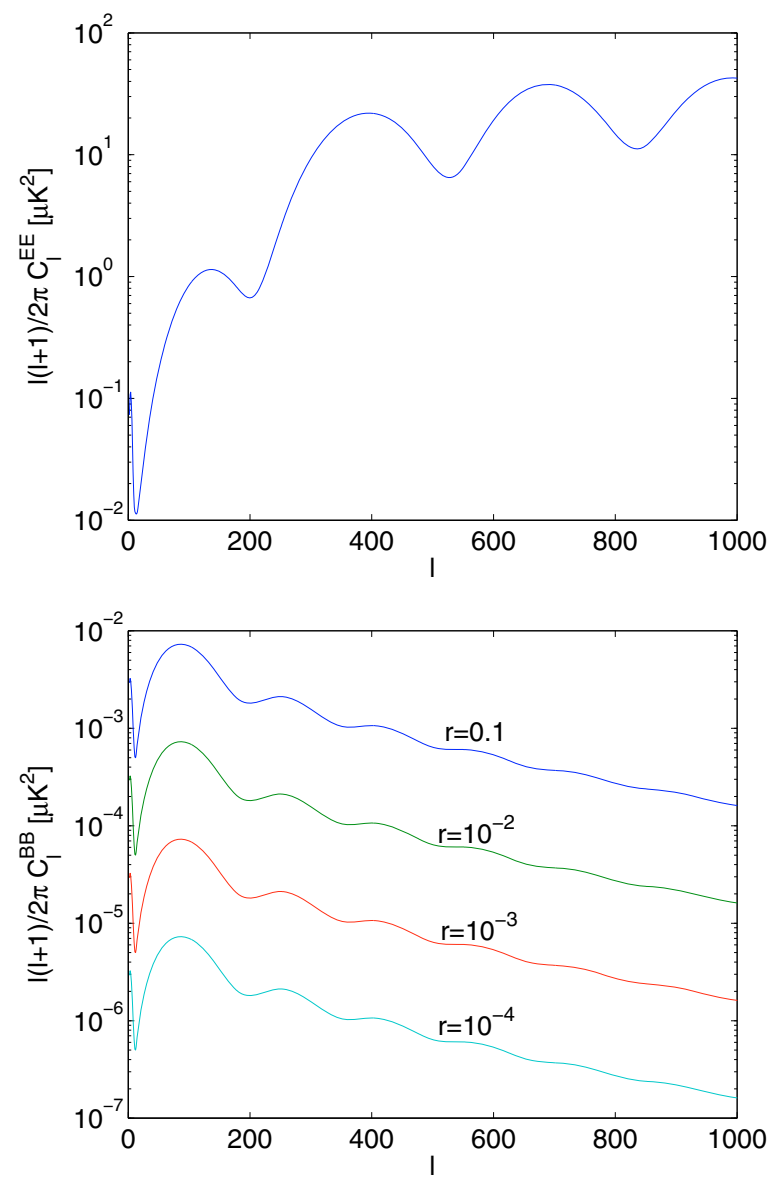

Fig. 1. The power spectrum of E (top) and B (bottom): no lensing, $\mathrm{B}$ mode power spectrum is plotted for various tensor-to-scalar ratio $r$.

show Eqs. (4) and (5) are equivalently given by:

$$
\begin{aligned}
& E(\hat{\boldsymbol{n}})=\sum \sqrt{\frac{(l+2) !}{(l-2) !}} a_{\mathrm{E}, l m} Y_{l m}(\hat{\boldsymbol{n}}) \\
& =\sum \sqrt{\frac{(l+2) !}{(l-2) !}} a_{\mathrm{E}, l m}^{*} Y_{l m}^{*}(\hat{\boldsymbol{n}}), \\
& =-\frac{1}{2}\left(\int F_{+}\left(\hat{\boldsymbol{n}^{\prime}}, \hat{\boldsymbol{n}}\right)\left[Q\left(\hat{\boldsymbol{n}}^{\prime}\right)-\mathrm{i} U\left(\hat{\boldsymbol{n}}^{\prime}\right)\right] \mathrm{d} \Omega^{\prime}\right. \\
& \left.+\int F_{-}\left(\hat{\boldsymbol{n}^{\prime}}, \hat{\boldsymbol{n}}\right)\left[Q\left(\hat{\boldsymbol{n}}^{\prime}\right)+\mathrm{i} U\left(\hat{\boldsymbol{n}^{\prime}}\right)\right] \mathrm{d} \Omega^{\prime}\right), \\
& B(\hat{\boldsymbol{n}})=\sum \sqrt{\frac{(l+2) !}{(l-2) !}} a_{\mathrm{B}, l m} Y_{l m}(\hat{\boldsymbol{n}}), \\
& =\sum \sqrt{\frac{(l+2) !}{(l-2) !}} a_{\mathrm{B}, l m}^{*} Y_{l m}^{*}(\hat{\boldsymbol{n}}), \\
& =\frac{\mathrm{i}}{2} \iint F_{+}\left(\hat{\boldsymbol{n}^{\prime}}, \hat{\boldsymbol{n}}\right)\left[Q\left(\hat{\boldsymbol{n}}^{\prime}\right)-\mathrm{i} U\left(\hat{\boldsymbol{n}^{\prime}}\right)\right] \mathrm{d} \Omega^{\prime} \\
& \left.-\int F_{-}\left(\hat{\boldsymbol{n}}^{\prime}, \hat{\boldsymbol{n}}\right)\left[Q\left(\hat{\boldsymbol{n}}^{\prime}\right)+\mathrm{i} U\left(\hat{\boldsymbol{n}}^{\prime}\right)\right] \mathrm{d} \Omega^{\prime}\right),
\end{aligned}
$$

where

$F_{ \pm}\left(\hat{\boldsymbol{n}^{\prime}}, \hat{\boldsymbol{n}}\right)=\sum_{l m} \sqrt{\frac{(l+2) !}{(l-2) !}}{ }_{2} Y_{l m}\left(\hat{\boldsymbol{n}}^{\prime}\right) Y_{l m}^{*}(\hat{\boldsymbol{n}})$. 

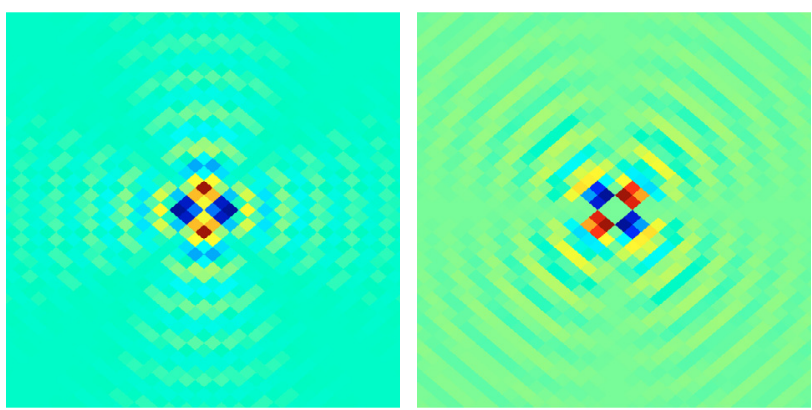

Fig. 2. Filter function: $\operatorname{Re}\left[F_{+}\left(\hat{\boldsymbol{n}}^{\prime}, \hat{\boldsymbol{n}}\right)\right]$ (left), $\operatorname{Im}\left[F_{+}\left(\hat{\boldsymbol{n}}^{\prime}, \hat{\boldsymbol{n}}\right)\right]$ (right) for a fixed $\boldsymbol{n}$, and $\boldsymbol{n}^{\prime}$ spanning $2^{\circ} \times 2^{\circ}$ area, $F_{+}\left(\hat{\boldsymbol{n}}^{\prime}, \hat{\boldsymbol{n}}\right)=$ $\sum_{l m}^{l \leq 1024} \sqrt{\frac{(l+2) !}{(l-2) !}} 2 Y_{l m}\left(\hat{\boldsymbol{n}}^{\prime}\right) Y_{l m}^{*}(\hat{\boldsymbol{n}})$.

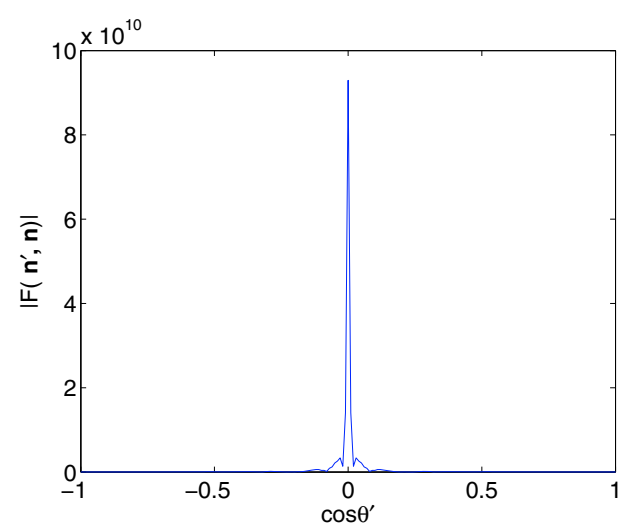

Fig. 3. Filter function: modulus $\left|F_{ \pm}\left(\hat{\boldsymbol{n}}^{\prime}, \hat{\boldsymbol{n}}\right)\right|$ for $(\theta=\pi / 2, \phi=0)$, highly peaked at $\cos \theta^{\prime}=0$.

Therefore, we may identify $F_{ \pm}\left(\hat{\boldsymbol{n}}^{\prime}, \hat{\boldsymbol{n}}\right)$ as pixel-space filters for $\mathrm{E} / \mathrm{B}$ decomposition. Using the property of spin- $s$ spherical harmonics ${ }_{s} Y_{l m}^{*}(\hat{\boldsymbol{n}})={ }_{-s} Y_{l-m}(\hat{\boldsymbol{n}})$, we may show the pair of the filter functions have complex conjugate relation:

$F_{+}\left(\hat{\boldsymbol{n}^{\prime}}, \hat{\boldsymbol{n}}\right)=F_{-}^{*}\left(\hat{\boldsymbol{n}^{\prime}}, \hat{\boldsymbol{n}}\right)$.

In Fig. 2, we show the real and imaginary part of $F_{+}\left(\hat{\boldsymbol{n}^{\prime}}, \hat{\boldsymbol{n}}\right)$ for a fixed $\boldsymbol{n}$. In Fig. 3, we show one dimensional plot of $\left|F_{ \pm}\left(\hat{\boldsymbol{n}}^{\prime}, \hat{\boldsymbol{n}}\right)\right|$ for a fixed $\boldsymbol{n}$. As shown in Figs. 2 and 3, our pixel-space filter possesses sharp peaks around $\hat{\boldsymbol{n}}$. Note that Eq. (12) would approach $\delta\left(\hat{\boldsymbol{n}}^{\prime}-\hat{\boldsymbol{n}}\right)$, if $\sqrt{\frac{(l+2) !}{(l-2) !}}{ }_{ \pm 2} Y_{l m}\left(\hat{\boldsymbol{n}}^{\prime}\right)$ were $Y_{l m}\left(\hat{\boldsymbol{n}}^{\prime}\right)$. Using Eq. (13), we may easily show Eqs. (10) and (11) are equivalently given by:

$$
\begin{aligned}
E(\hat{\boldsymbol{n}}) & =-\int \mathrm{d} \Omega^{\prime} \operatorname{Re}\left[F_{+}\left(\hat{\boldsymbol{n}^{\prime}}, \hat{\boldsymbol{n}}\right)\left(Q\left(\hat{\boldsymbol{n}}^{\prime}\right)-\mathrm{i} U\left(\hat{\boldsymbol{n}^{\prime}}\right)\right)\right], \\
& =-\int \mathrm{d} \Omega^{\prime} \operatorname{Re}\left[F_{-}\left(\hat{\boldsymbol{n}^{\prime}}, \hat{\boldsymbol{n}}\right)\left(Q\left(\hat{\boldsymbol{n}}^{\prime}\right)+\mathrm{i} U\left(\hat{\boldsymbol{n}}^{\prime}\right)\right)\right] . \\
B(\hat{\boldsymbol{n}}) & =-\int \mathrm{d} \Omega^{\prime} \operatorname{Im}\left[F_{+}\left(\hat{\boldsymbol{n}^{\prime}}, \hat{\boldsymbol{n}}\right)\left(Q\left(\hat{\boldsymbol{n}}^{\prime}\right)-\mathrm{i} U\left(\hat{\boldsymbol{n}}^{\prime}\right)\right)\right], \\
& =\int \mathrm{d} \Omega^{\prime} \operatorname{Im}\left[F_{-}\left(\hat{\boldsymbol{n}^{\prime}}, \hat{\boldsymbol{n}}\right)\left(Q\left(\hat{\boldsymbol{n}^{\prime}}\right)+\mathrm{i} U\left(\hat{\boldsymbol{n}}^{\prime}\right)\right)\right] .
\end{aligned}
$$

\section{Incomplete sky coverage}

Due to heavy foreground contamination, CMB polarization signal is not estimated reliably over a whole sky. For instance, the WMAP team have subtracted diffuse foregrounds by templatefitting, and masked the regions that cannot be cleaned reliably. In
Fig. 7, we show a foreground mask, which combines the WMAP team's polarization mask with the point source mask and shall be used for our simulation. The E/B decomposition coefficients from a masked sky are given by:

$\tilde{a}_{\mathrm{E}, l m}=-\left(\tilde{a}_{2, l m}+\tilde{a}_{-2, l m}\right) / 2$,

$\tilde{a}_{\mathrm{B}, l m}=\mathrm{i}\left(\tilde{a}_{2, l m}-\tilde{a}_{-2, l m}\right) / 2$,

where

$\tilde{a}_{ \pm 2, l m}=\int W\left(\hat{\boldsymbol{n}}^{\prime}\right)[Q(\hat{\boldsymbol{n}}) \pm \mathrm{i} U(\hat{\boldsymbol{n}})]_{ \pm 2} Y_{l m}^{*}(\hat{\boldsymbol{n}}) \mathrm{d} \Omega$,

and $W\left(\hat{\boldsymbol{n}}^{\prime}\right)$ is a foreground mask. Therefore, E/B maps reconstructed from incomplete sky are given by:

$$
\begin{aligned}
& \tilde{E}(\hat{\boldsymbol{n}})=\sqrt{\frac{(l+2) !}{(l-2) !}} \tilde{a}_{\mathrm{E}, l m} Y_{l m}(\hat{\boldsymbol{n}}), \\
& =-\frac{1}{2}\left(\int W\left(\hat{\boldsymbol{n}^{\prime}}\right) F_{+}\left(\hat{\boldsymbol{n}^{\prime}}, \hat{\boldsymbol{n}}\right)\left[Q\left(\hat{\boldsymbol{n}}^{\prime}\right)-\mathrm{i} U\left(\hat{\boldsymbol{n}^{\prime}}\right)\right] \mathrm{d} \Omega^{\prime}\right. \\
& \left.+\int W\left(\hat{\boldsymbol{n}^{\prime}}\right) F_{-}\left(\hat{\boldsymbol{n}^{\prime}}, \hat{\boldsymbol{n}}\right)\left[Q\left(\hat{\boldsymbol{n}}^{\prime}\right)+\mathrm{i} U\left(\hat{\boldsymbol{n}^{\prime}}\right)\right] \mathrm{d} \Omega^{\prime}\right), \\
& \tilde{B}(\hat{\boldsymbol{n}})=\sqrt{\frac{(l+2) !}{(l-2) !}} \tilde{a}_{\mathrm{B}, l m} Y_{l m}(\hat{\boldsymbol{n}}), \\
& =\frac{\mathrm{i}}{2}\left(\int W\left(\hat{\boldsymbol{n}^{\prime}}\right) F_{+}\left(\hat{\boldsymbol{n}^{\prime}}, \hat{\boldsymbol{n}}\right)\left[Q\left(\hat{\boldsymbol{n}^{\prime}}\right)-\mathrm{i} U\left(\hat{\boldsymbol{n}^{\prime}}\right)\right] \mathrm{d} \Omega^{\prime}\right. \\
& \left.-\int W\left(\hat{\boldsymbol{n}}^{\prime}\right) F_{-}\left(\hat{\boldsymbol{n}^{\prime}}, \hat{\boldsymbol{n}}\right)\left[Q\left(\hat{\boldsymbol{n}}^{\prime}\right)+\mathrm{i} U\left(\hat{\boldsymbol{n}}^{\prime}\right)\right] \mathrm{d} \Omega^{\prime}\right) .
\end{aligned}
$$

Since filter functions $F_{ \pm}\left(\hat{\boldsymbol{n}^{\prime}}, \hat{\boldsymbol{n}}\right)$ are sharply peaked around $\hat{\boldsymbol{n}}$, certain pixels far away from masked regions may contain negligible $\mathrm{E} / \mathrm{B}$ mixing, and vice versa. Equivalently, E/B mixing is localized in pixels close to the masked regions. For higher pixel resolution, Eq. (12) contains summation up to higher $l$, which makes the peak of the filter function sharper. Therefore, E/B mixing decreases with increase in pixel resolution. The spherical harmonic method (the first line of Eqs. (17) and (18)) are much faster than the pixel-space method (the second line), while they are mathematically equivalent. Therefore, we are going to rely on spherical harmonic transformation method for our simulation in the next section. However, it should be kept in mind that the pixel-space approach have provided useful insights on E/B decomposition of incomplete sky.

Using Eq. (18), we may show the expected power of $\tilde{B}(\hat{\boldsymbol{n}})$ is given by:

$$
\begin{aligned}
& \left\langle\tilde{B}^{2}(\hat{\boldsymbol{n}})\right\rangle=\frac{1}{4} \int \mathrm{d} \Omega^{\prime} \mathrm{d} \Omega^{\prime \prime} W\left(\hat{\boldsymbol{n}^{\prime}}\right) W\left(\hat{\boldsymbol{n}^{\prime \prime}}\right) \\
& \quad \times\left(F_{+}\left(\hat{\boldsymbol{n}^{\prime}}, \hat{\boldsymbol{n}}\right) F_{-}\left(\hat{\boldsymbol{n}^{\prime \prime}}, \hat{\boldsymbol{n}}\right)\left\langle\left(Q\left(\hat{\boldsymbol{n}^{\prime}}\right)-\mathrm{i} U\left(\hat{\boldsymbol{n}^{\prime}}\right)\right)\left(Q\left(\hat{\boldsymbol{n}^{\prime \prime}}\right)+\mathrm{i} U\left(\hat{\boldsymbol{n}^{\prime \prime}}\right)\right)\right\rangle\right. \\
& \quad+F_{-}\left(\hat{\boldsymbol{n}^{\prime}}, \hat{\boldsymbol{n}}\right) F_{+}\left(\hat{\boldsymbol{n}^{\prime \prime}}, \hat{\boldsymbol{n}}\right)\left\langle\left(Q\left(\hat{\boldsymbol{n}^{\prime}}\right)+\mathrm{i} U\left(\hat{\boldsymbol{n}^{\prime}}\right)\right)\left(Q\left(\hat{\boldsymbol{n}^{\prime \prime}}\right)-\mathrm{i} U\left(\hat{\boldsymbol{n}^{\prime \prime}}\right)\right)\right\rangle \\
& \quad-F_{+}\left(\hat{\boldsymbol{n}^{\prime}}, \hat{\boldsymbol{n}}\right) F_{+}\left(\hat{\boldsymbol{n}^{\prime \prime}}, \hat{\boldsymbol{n}}\right)\left\langle\left(Q\left(\hat{\boldsymbol{n}^{\prime}}\right)-\mathrm{i} U\left(\hat{\hat{n}^{\prime}}\right)\right)\left(Q\left(\hat{\boldsymbol{n}^{\prime \prime}}\right)-\mathrm{i} U\left(\hat{\boldsymbol{n}^{\prime \prime}}\right)\right)\right\rangle \\
& \left.\quad-F_{-}\left(\hat{\boldsymbol{n}^{\prime}}, \hat{\boldsymbol{n}}\right) F_{-}\left(\hat{\boldsymbol{n}^{\prime \prime}}, \hat{\boldsymbol{n}}\right)\left\langle\left(Q\left(\hat{\boldsymbol{n}^{\prime}}\right)+\mathrm{i} U\left(\hat{\boldsymbol{n}^{\prime}}\right)\right)\left(Q\left(\hat{\boldsymbol{n}^{\prime \prime}}\right)+\mathrm{i} U\left(\hat{\boldsymbol{n}^{\prime \prime}}\right)\right)\right\rangle\right),
\end{aligned}
$$

where $\langle\ldots\rangle$ denotes an ensemble average, and

$$
\begin{gathered}
\left\langle\left(Q\left(\hat{\boldsymbol{n}^{\prime}}\right)+\mathrm{i} U\left(\hat{\boldsymbol{n}^{\prime}}\right)\right)\left(Q\left(\hat{\boldsymbol{n}^{\prime \prime}}\right)+\mathrm{i} U\left(\hat{\boldsymbol{n}^{\prime \prime}}\right)\right)\right\rangle= \\
\sum_{l} \sqrt{\frac{2 l+1}{4 \pi}}\left(C_{l}^{\mathrm{EE}}+C_{l}^{\mathrm{BB}}\right)_{2} Y_{l,-2}(\beta, 0) \mathrm{e}^{2 \mathrm{i}(\alpha+\gamma)},
\end{gathered}
$$




$$
\begin{gathered}
\left\langle\left(Q\left(\hat{\boldsymbol{n}}^{\prime}\right) \mp \mathrm{i} U\left(\hat{\boldsymbol{n}}^{\prime}\right)\right)\left(Q\left(\hat{\boldsymbol{n}^{\prime \prime}}\right) \pm \mathrm{i} U\left(\hat{\boldsymbol{n}^{\prime \prime}}\right)\right)\right\rangle= \\
\sum_{l} \sqrt{\frac{2 l+1}{4 \pi}}\left(C_{l}^{\mathrm{EE}}+C_{l}^{\mathrm{BB}}\right)_{2} Y_{l,-2}(\beta, 0) \mathrm{e}^{ \pm \mathrm{i}(\alpha-\gamma)},
\end{gathered}
$$

and $\beta$ is the separation angle between $\hat{n}^{\prime}$ and $\hat{n}^{\prime \prime}, \alpha$ and $\gamma$ are the rotation angles respectively, which align $\hat{e}_{\theta}$ at $\hat{n}^{\prime}$ and $\hat{n}^{\prime \prime}$ with the great circle passing through $\hat{n}^{\prime}$ and $\hat{n}^{\prime \prime}$ (refer to Fig. 1 of $\mathrm{Ng}$ \& Liu 1999, for a geometrical diagram). Taking into account Eqs. (19)-(21), we may easily show that the expected leakage power at $\hat{\boldsymbol{n}}$ is given by

$$
\begin{aligned}
& \left\langle\tilde{B}_{\mathrm{E}}^{2}(\hat{\boldsymbol{n}})\right\rangle=\frac{1}{4} \int \mathrm{d} \Omega^{\prime} \mathrm{d} \Omega^{\prime \prime} W\left(\hat{\boldsymbol{n}^{\prime}}\right) W\left(\hat{\boldsymbol{n}^{\prime \prime}}\right) \\
& \times\left(F_{+}\left(\hat{\boldsymbol{n}^{\prime}}, \hat{\boldsymbol{n}}\right) F_{-}\left(\hat{\boldsymbol{n}^{\prime \prime}}, \hat{\boldsymbol{n}}\right)\left\langle\left(Q_{\mathrm{E}}\left(\hat{\boldsymbol{n}^{\prime}}\right)-\mathrm{i} U_{\mathrm{E}}\left(\hat{\boldsymbol{n}^{\prime}}\right)\right)\left(Q_{\mathrm{E}}\left(\hat{\boldsymbol{n}^{\prime \prime}}\right)+\mathrm{i} U_{\mathrm{E}}\left(\hat{\boldsymbol{n}^{\prime \prime}}\right)\right)\right\rangle\right. \\
& +F_{-}\left(\hat{\boldsymbol{n}^{\prime}}, \hat{\boldsymbol{n}}\right) F_{+}\left(\hat{\boldsymbol{n}^{\prime \prime}}, \hat{\boldsymbol{n}}\right)\left\langle\left(Q_{\mathrm{E}}\left(\hat{\boldsymbol{n}^{\prime}}\right)+\mathrm{i} U_{\mathrm{E}}\left(\hat{\boldsymbol{n}^{\prime}}\right)\right)\left(Q_{\mathrm{E}}\left(\hat{\boldsymbol{n}^{\prime \prime}}\right)-\mathrm{i} U_{\mathrm{E}}\left(\hat{\boldsymbol{n}^{\prime \prime}}\right)\right)\right\rangle \\
& -F_{+}\left(\hat{\boldsymbol{n}^{\prime}}, \hat{\boldsymbol{n}}\right) F_{+}\left(\hat{\boldsymbol{n}^{\prime \prime}}, \hat{\boldsymbol{n}}\right)\left\langle\left(Q_{\mathrm{E}}\left(\hat{\boldsymbol{n}^{\prime}}\right)-\mathrm{i} U_{\mathrm{E}}\left(\hat{\boldsymbol{n}^{\prime}}\right)\right)\left(Q_{\mathrm{E}}\left(\hat{\boldsymbol{n}^{\prime \prime}}\right)-\mathrm{i} U_{\mathrm{E}}\left(\hat{\boldsymbol{n}^{\prime \prime}}\right)\right)\right\rangle \\
& \left.-F_{-}\left(\hat{\boldsymbol{n}^{\prime}}, \hat{\boldsymbol{n}}\right) F_{-}\left(\hat{\boldsymbol{n}^{\prime \prime}}, \hat{\boldsymbol{n}}\right)\left\langle\left(Q_{\mathrm{E}}\left(\hat{\boldsymbol{n}^{\prime}}\right)+\mathrm{i} U_{\mathrm{E}}\left(\hat{\boldsymbol{n}^{\prime}}\right)\right)\left(Q_{\mathrm{E}}\left(\hat{\boldsymbol{n}^{\prime \prime}}\right)+\mathrm{i} U_{\mathrm{E}}\left(\hat{\boldsymbol{n}^{\prime \prime}}\right)\right)\right\rangle\right),
\end{aligned}
$$

where

$$
\begin{aligned}
& \left\langle\left(Q_{\mathrm{E}}\left(\hat{\boldsymbol{n}^{\prime}}\right)+\mathrm{i} U_{\mathrm{E}}\left(\hat{\boldsymbol{n}^{\prime}}\right)\right)\left(Q_{\mathrm{E}}\left(\hat{\boldsymbol{n}^{\prime \prime}}\right)+\mathrm{i} U_{\mathrm{E}}\left(\hat{\boldsymbol{n}^{\prime \prime}}\right)\right)\right\rangle= \\
& \sum_{l} \sqrt{\frac{2 l+1}{4 \pi}} C_{l}^{\mathrm{EE}}{ }_{2} Y_{l,-2}(\beta, 0) \mathrm{e}^{2 \mathrm{i}(\alpha+\gamma)}, \\
& \left\langle\left(Q_{\mathrm{E}}\left(\hat{\boldsymbol{n}^{\prime}}\right) \mp \mathrm{i} U_{\mathrm{E}}\left(\hat{\boldsymbol{n}^{\prime}}\right)\right)\left(Q_{\mathrm{E}}\left(\hat{\boldsymbol{n}^{\prime \prime}}\right) \pm \mathrm{i} U_{\mathrm{E}}\left(\hat{\boldsymbol{n}^{\prime \prime}}\right)\right)\right\rangle= \\
& \sum_{l} \sqrt{\frac{2 l+1}{4 \pi}} C_{l}^{\mathrm{EE}}{ }_{2} Y_{l,-2}(\beta, 0) \mathrm{e}^{ \pm 2 \mathrm{i}(\alpha-\gamma)} .
\end{aligned}
$$

Therefore, we may diagnose ambiguous pixels (i.e. heavy $\mathrm{E} / \mathrm{B}$ mixing) by comparing $\left\langle\tilde{B}_{\mathrm{E}}^{2}(\hat{\boldsymbol{n}})\right\rangle$ with $\left\langle\tilde{B}_{\mathrm{B}}^{2}(\hat{\boldsymbol{n}})\right\rangle$, where $\left\langle\tilde{B}_{\mathrm{B}}^{2}(\hat{\boldsymbol{n}})\right\rangle$ is the local power contributed by $\mathrm{B}$ mode and given by replacing "E" with "B" in Eqs. (22)-(24). However, estimating Eqs. (22)-(24) is prohibitively complicated. Therefore, we are going to resort to Monte-Carlo simulations in order to estimate $\left\langle\tilde{B}_{\mathrm{E}}^{2}(\hat{\boldsymbol{n}})\right\rangle /\left\langle\tilde{B}_{\mathrm{B}}^{2}(\hat{\boldsymbol{n}})\right\rangle$. Depending $\left\langle\tilde{B}_{\mathrm{E}}^{2}(\hat{\boldsymbol{n}})\right\rangle /\left\langle\tilde{B}_{\mathrm{B}}^{2}(\hat{\boldsymbol{n}})\right\rangle$, we may classify the pixel at $\hat{\boldsymbol{n}}$ as "pure" and "ambiguous". To be specific, we may retain pixels satisfying:

$\frac{\left\langle\tilde{B}_{\mathrm{E}}^{2}(\hat{\boldsymbol{n}})\right\rangle}{\left\langle\tilde{B}_{\mathrm{B}}^{2}(\hat{\boldsymbol{n}})\right\rangle}<\frac{r_{\mathrm{c}}}{r}$,

where $r$ is the assumed tensor-to-scalar ratio of Monte-Carlo simulation, from which $\left\langle\tilde{B}_{\mathrm{E}}^{2}(\hat{\boldsymbol{n}})\right\rangle /\left\langle\tilde{\boldsymbol{B}}_{\mathrm{B}}^{2}(\hat{\boldsymbol{n}})\right\rangle$ is estimated. Therefore, the level of leakage in retained pixels is comparable to the primordial B mode power spectrum of tensor-to-scalar ratio $r_{\mathrm{c}}$. In Fig. 4, we show the sky fraction for various $r_{\mathrm{c}}$, given a foreground mask shown in Fig. 7. Since sky fraction decreases with lower $r_{\mathrm{c}}$, we may not simply set $r_{\mathrm{c}}$ to a lowest value. Therefore, we need to derive an optimal $r_{\mathrm{c}}$, which minimizes the estimation error. The estimation error of $\mathrm{B}$ mode power spectrum is given by:

$$
\begin{aligned}
\Delta C_{l}^{\mathrm{BB}} & =\frac{2}{(2 l+1) f_{\mathrm{sky}}}\left(C_{l}^{\mathrm{BB}}+\tilde{C}_{l}^{\mathrm{EE}}+N_{l}\right), \\
& \approx \frac{2}{(2 l+1) f_{\mathrm{sky}}}\left(C_{l}^{\mathrm{BB}}+\frac{r_{\mathrm{c}}}{r} C_{l}^{\mathrm{BB}}+N_{l}\right),
\end{aligned}
$$

where $N_{l}$ is noise power spectrum. Note that the leakage does not bias the $\mathrm{B}$ mode power spectrum estimation, but increases the variance, when the power spectrum estimation is made by

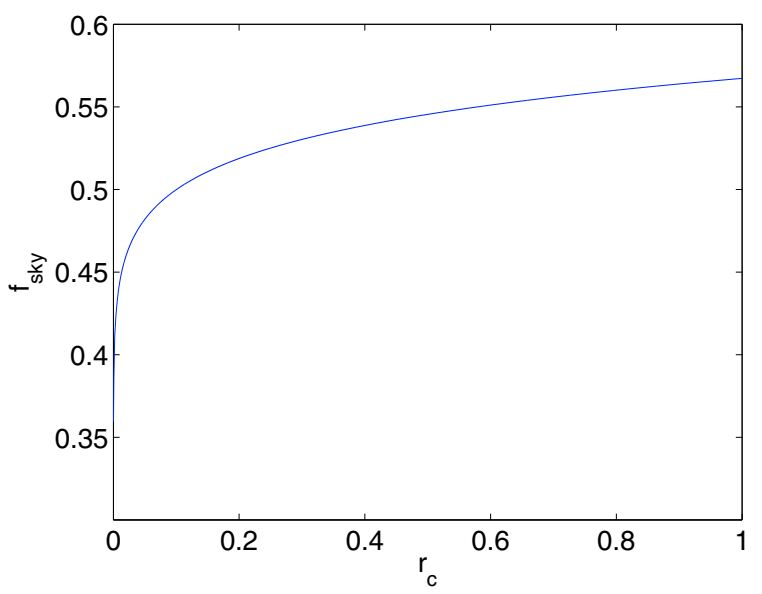

Fig. 4. Effective sky fraction $f_{\text {sky }}$ for various cut level $r_{\mathrm{c}}$.

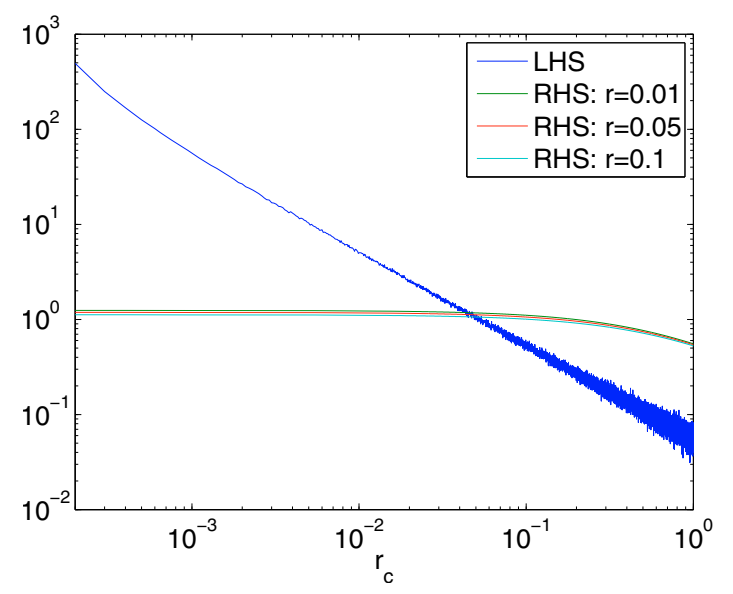

Fig. 5. Numerical solution of Eq. (27) for various $r$ and the noise level of Planck HFI instrument: two plots represent the left hand side (LHS) and right hand side (RHS) of Eq. (27).

a pseudo- $C_{l}$ method and leakage is taken care of (Hivon et al. 2002a; Grain et al. 2009). By requiring $\partial \Delta C_{l}^{\mathrm{BB}} / \partial r_{\mathrm{c}}=0$, we get

$\frac{\partial \ln f_{\text {sky }}}{\partial r_{\mathrm{c}}}=\frac{1}{r+r_{\mathrm{c}}+N_{l} / C_{l}^{\mathrm{BB}}(r=1)}$.

In Fig. 5, we plot the left and right hand side of Eq. (27) for the noise level of Planck HFI instrument, and the multipole $l=86$, which is the peak multipole of primordial $\mathrm{B}$ mode power spectrum. From Fig. 5, we find curves intersect at $r_{\mathrm{c}} \approx 4 \times 10^{-2}$ with weak dependence on $r$. It should be noted that the weak dependence is due to the low signal-to-noise ratio of the considered experiment (i.e. $N_{l} / C_{l}^{\mathrm{BB}}(r=1) \gg 0$ ), and the dependence on $r$ is not weak in general. We are going to use $r_{\mathrm{c}} \approx 4 \times 10^{-2}$ for the simulation in the next section.

\section{Application to simulated data}

Using the WMAP concordance $\Lambda$ CDM model, we have simulated Stokes parameter $Q$ and $U$ over a whole-sky with a HEALPix pixel resolution $\left(N_{\text {side }}=1024\right)$ and $10^{\prime} F W H M$ beam. We have made the inputmap to contain no B mode polarization. Therefore, any non-zero values in output B map are attributed to leakage. We show our simulated polarization map in Fig. 6, where the orientation and length of headless arrows indicates polarization angle and amplitude respectively. Note that the polarization map shows only gradient-like patterns, because they 


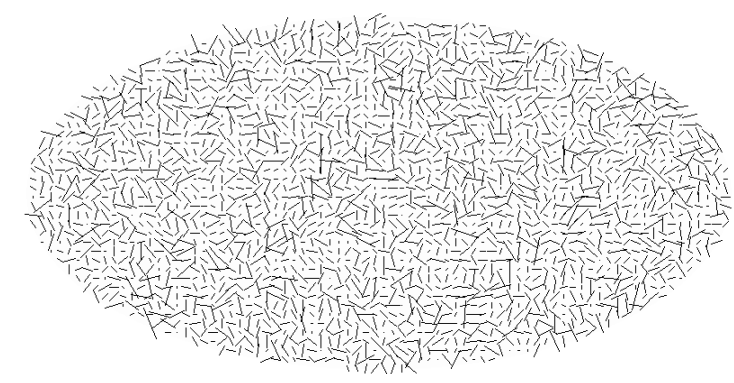

Fig. 6. Input polarization map: E mode polarization only.

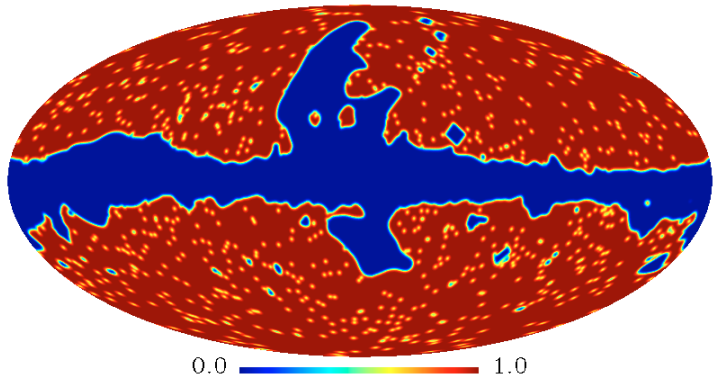

Fig. 7. Foreground mask: smoothed with $1.5^{\circ}$ FWHM Gaussian kernel.

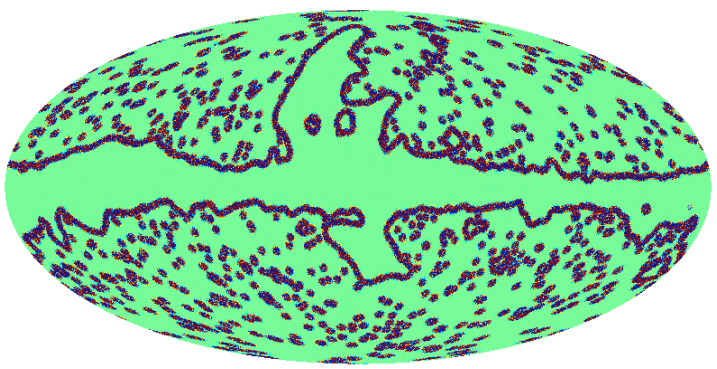

Fig. 8. Output $\tilde{B}$ map from incomplete sky.

contain only E mode polarization. It is well-known that $\mathrm{E} / \mathrm{B}$ mixing increases with the length of cut sky boundary (Bunn et al. 2003). We have combined the WMAP team's polarization mask with the point source mask, and prograded it to $N_{\text {side }}=1024$. In order to reduce sharp boundaries, we have smoothed the mask with $1.5^{\circ} \mathrm{FWHM}$ Gaussian kernel. We have referred to the WMAP team's boundary smoothing process of Internal Linear Combination map (Hinshaw et al. 2007). Nevertheless, it should be noted that smoothed boundary is not essential to our method, and further improvement may be possible by using more sophisticated smoothing kernel (Das et al. 2009). In Fig. 7, we show our smoothed mask, whose sky fraction amounts to 0.71 .

In Fig. 8, we show a $\tilde{B}$ map, which we have produced from the masked polarization map. Using $\left\langle\tilde{B}_{\mathrm{E}}^{2}(\hat{\boldsymbol{n}})\right\rangle /\left\langle\tilde{B}_{\mathrm{B}}^{2}(\hat{\boldsymbol{n}})\right\rangle$ estimated from $10^{3}$ simulation, we have diagnosed ambiguous pixels, and retained pixels of $\left\langle\tilde{B}_{\mathrm{E}}^{2}(\hat{\boldsymbol{n}})\right\rangle /\left\langle\tilde{B}_{\mathrm{B}}^{2}(\hat{\boldsymbol{n}})\right\rangle<4 \times 10^{-2} / r$. In Fig. 9, we show the $\mathrm{B}$ map, where ambiguous pixels are excluded. We find retained pixels of Fig. 9 amount to sky fraction $f_{\text {sky }}=0.48$. From the retained pixels, we have estimated the leakage power spectrum by pseudo $C_{l}$ method (Wandelt et al. 2001; Hivon et al. 2002 b). Power spectrum is usually estimated by a pseudo $C_{l}$ method at high multipoles $(l>30)$, while by maximum likelihood method or Gibbs sampling at low multipoles $(l \leq 30)$ (Bond et al. 1998; Eriksen et al. 2004; Hinshaw et al. 2007; Efstathiou 2006). However, we find pseudo $C_{l}$ method at low multipoles is good enough for our need, since we do not intend accurate estimation of likelihood function. Besides that, we

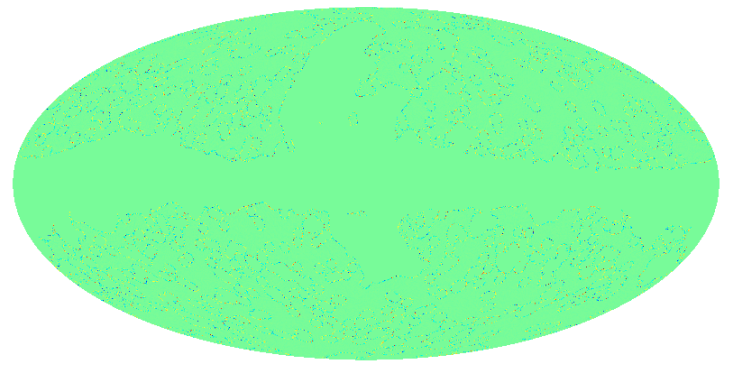

Fig. 9. Filerted $\tilde{B}$ map: masked by a foreground mask, and pixels of $\left\langle\tilde{\boldsymbol{B}}_{\mathrm{E}}^{2}(\hat{\boldsymbol{n}})\right\rangle /\left\langle\tilde{\boldsymbol{B}}_{\mathrm{B}}^{2}(\hat{\boldsymbol{n}})\right\rangle>4 \times 10^{-2}$ are set to zero.

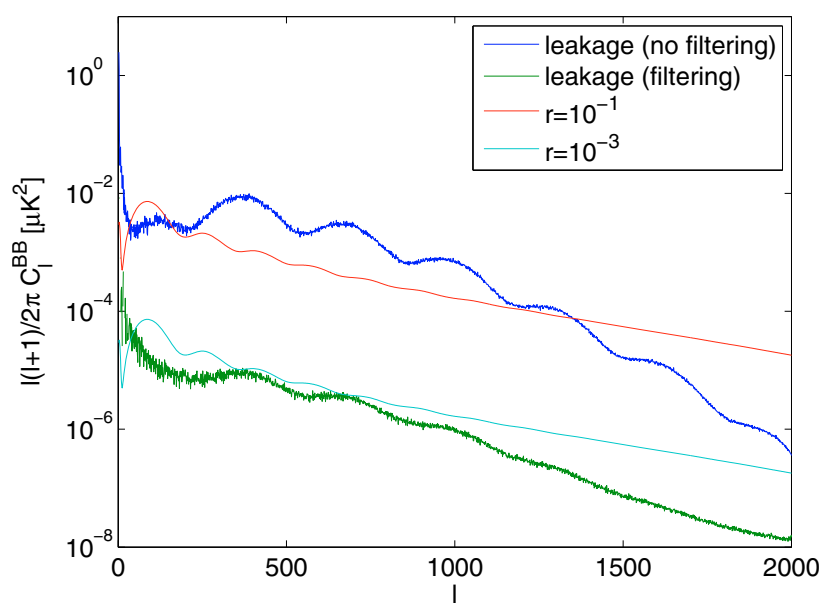

Fig. 10. Leakage power spectrum and primordial B mode power of various tensor-to-scalar ratio $r$ : a blue curve correspond to the leakage power estimated without ambiguous pixel filtering (Fig. 8), a green curve to the leakage power estimated with ambiguous pixel filtering (Fig. 9).

are mainly interested in leakage power at $(l>30)$, because primordial B mode power spectrum has a peak around multipoles $l \sim 90$ (see Fig. 1). In Fig. 10, we show the leakage power spectrum and B mode power spectrum of various tensor-to-scalar ratio $r$. Fig. 10 shows leakage power (green) is smaller than B mode power spectrum of $r=10^{-3}$ at wide range of multipoles $(50 \lesssim l \lesssim 2000$ ), when ambiguous pixels are excluded (i.e. the B map in Fig. 9). In Fig. 10, we also show the leakage power (blue) estimated without filtering (i.e. the B map in Fig. 8). Obviously, we have reduced leakage significantly by excluding ambiguous pixels.

\section{Scale-dependence of leakage}

In order to reduce leakage, we have removed ambiguous pixels in a scale-independent way. On the other hand, it is known that leakage has some dependence on scales as well as realspace. Specifically, leakage of low $l$ extends over large area, while leakage of high $l$ is often confined to the small area nearby the boundary. Therefore, one may argue that our method does not reduce the leakage at low $l$ as effectively as that of high $l$ or leads to unnecessary loss of information. However, as shown in Fig. 8, leakage is relatively localized in pixel space, and we were able to reduced leakage very significantly at wide range of multipoles, while retaining sky fraction 0.48 . Besides that, our simulation shows the leakage at lowest $l$ is reduced significantly as well. It is also possible to implement a further leakage reduction in a scale-dependent way, after ambiguous pixels are removed. Nevertheless, a hybrid method, which exploits scale 
and position dependence simultaneously, may be most optimal. A wavelet approach may be promising for such implementation, since wavelet functions are, in general, well-localized in harmonic space as well as pixel space. We defer a rigorous investigation to a separate publication.

\section{Discussion}

We have investigated $\mathrm{E} / \mathrm{B}$ decomposition in pixel space, and shown that we may produce $\mathrm{E} / \mathrm{B}$ decomposed maps by convolving polarization maps with certain filter functions of a sharp peak. We find that $\mathrm{E} / \mathrm{B}$ mixing due to incomplete sky is localized in pixel-space, and negligible in the regions far away from masked area. By estimating the expected local leakage power and comparing it with the expected pure mode power, we have diagnosed ambiguous pixels and excluded them. Our criteria for ambiguous pixels (i.e. $r_{\mathrm{c}}$ ) is associated with the tensor-to-scalar ratio of $\mathrm{B}$ mode power spectrum, which the leakage power is comparable to. The estimation error $\Delta C_{l}$ may increases with lower $r_{\mathrm{c}}$, because sky fraction decreases. Therefore, we have solved $\partial \Delta C_{l} / \partial r_{\mathrm{c}}=0$ and obtained the optimal $r_{\mathrm{c}}$, which minimizes the estimation error, given a foreground mask and noise level. We have applied our method to simulated maps blocked by a foreground mask. Simulation shows that leakage power is subdominant in comparison with unlensed B mode power spectrum of $r \sim 1 \times 10^{-3}$ at wide range of multipoles $(50 \lesssim l \lesssim 2000)$, while pixels of sky fraction 0.48 are retained. We may apply our method equally to small sky patch observation, by treating unobserved sky as masked region. From simulation with sky patch of simple symmetric shape, we have confirmed our method reduce $\mathrm{E} / \mathrm{B}$ mixing very effectively. A rigorous investigation is deferred to a separate publication.

Noise is slightly correlated from pixel to pixel in E and $\mathrm{B}$ maps, even when interpixel correlation is absent in $\mathrm{Q}$ and $\mathrm{U}$ maps. However, this interpixel noise correlation induced by $\mathrm{E} / \mathrm{B}$ decomposition is not confined to our method, but $\mathrm{E} / \mathrm{B}$ decomposition in general. Besides that, we find interpixel noise correlation may be neglected without sacrificing the accuracy of error analysis (refer to Appendix A for details). Therefore, it does not limit the applicability of our method.

Current observations such as WMAP were unable to detect B mode polarization. Therefore, we did not attempt to apply our method to observation data. When Planck polarization data of high Signal-to-Noise-Ratio (SNR) are available in near future, we may apply our method to the data, and be able to detect B mode polarization.

Acknowledgements. We are grateful to the anonymous referee for thorough reading and helpful comments, which leads to significant improvement of this work. We acknowledge the use of the Legacy Archive for Microwave Background Data Analysis (LAMBDA) and the HEALPix package (Gorski et al. 1999; Gorski et al. 2005). This work is supported by FNU grant 272-06-0417, 272-07-0528 and 21-04-0355. This work is supported in part by Danmarks Grundforskningsfond, which allowed the establishment of the Danish Discovery Center.

\section{Appendix A: Error analysis}

Power spectrum is usually estimated by pseudo- $C_{l}$ method at high multipoles (Wandelt et al. 2001; Hivon et al. 2002b; Efstathiou 2006; Nolta et al. 2009; Larson et al. 2010). According to pseudo- $C_{l}$ method, we may estimate power spectrum as follows (Wandelt et al. 2001; Hivon et al. 2002b):

$\hat{C}_{l}^{\mathrm{BB}}=\sum_{l^{\prime}}\left(\boldsymbol{M}^{-1}\right)_{l l^{\prime}} \tilde{C}_{l^{\prime}}^{\mathrm{BB}}$, where

$\boldsymbol{M}_{l l^{\prime}}=\frac{2 l^{\prime}+1}{4 \pi} \sum_{l^{\prime \prime} m^{\prime \prime}}\left|w_{l^{\prime \prime} m^{\prime \prime}}\right|^{2}\left\{\left(\begin{array}{ccc}l & l^{\prime} & l^{\prime \prime} \\ 2 & 0 & -2\end{array}\right)-\left(\begin{array}{ccc}l & l^{\prime} & l^{\prime \prime} \\ -2 & 0 & 2\end{array}\right)\right\}^{2}$

and

$w_{l^{\prime \prime} m^{\prime \prime}}=\int \mathrm{d} \Omega W(\hat{\boldsymbol{n}}) Y_{l^{\prime \prime} m^{\prime \prime}}^{*}(\hat{\boldsymbol{n}})$,

with $W(\hat{\boldsymbol{n}})$ being a foreground mask function. The pseudoquantity $\tilde{C}_{l}$ and $\tilde{a}_{l m}^{j}$ are given by:

$\tilde{C}_{l}=\frac{1}{2 l+1} \sum_{m} \tilde{a}_{l m}^{j}\left(\tilde{a}_{l m}^{k}\right)^{*}$

$\tilde{a}_{l m}^{j}=\int \mathrm{d} \Omega W(\hat{\boldsymbol{n}}) \Delta^{j}(\hat{\boldsymbol{n}}) Y_{l m}^{*}(\hat{\boldsymbol{n}})$,

where $j$ and $k$ refers to a DA-year combination and $\Delta(\hat{\boldsymbol{n}})$ refers to data (T, E or B). We may split $\tilde{a}_{l m}^{j}$ into signal and noise:

$\tilde{a}_{l m}^{j}=\tilde{a}_{l m}+\tilde{N}_{l m}^{j}$

The noise part is given by:

$\tilde{N}_{l m}=\frac{4 \pi}{n_{\mathrm{pix}}} \sum_{i} N_{i} Y_{l m}^{i}$

where a pixel index $i$ runs over pixels outside a foreground mask, and $N_{i}$ refers to noise at $i$ th pixel. By central limit theorem (Arfken \& Weber 2000; Riley et al. 2006), $\tilde{N}_{l m}^{j}$ follows a Gaussian distribution, and is uncorrelated among distinct $j$ th DA-year data (i.e. $\left.\left\langle\tilde{N}_{l m}^{j}\left(\tilde{N}_{l m}^{k}\right)^{*}\right\rangle \propto \delta_{j k}\right)$.

If cross power spectra are used (i.e. $j \neq k$ ), noise does not bias estimation, and its statistical properties need to be known only for errors analysis (Hinshaw et al. 2007). In order to understand the effect of noise on error analysis, let us consider covariance of $\hat{C}_{l}$ :

$\operatorname{Cov}\left(\hat{C}_{l_{1}}, \hat{C}_{l_{2}}\right)=\left\langle\hat{C}_{l_{1}} \hat{C}_{l_{2}}\right\rangle-C_{l_{1}} C_{l_{2}}$,

where $C_{l}$ is a theoretical power spectrum and

$$
\begin{aligned}
\left\langle\hat{C}_{l_{1}} \hat{C}_{l_{2}}\right\rangle & =\left\langle\sum_{l}\left(\boldsymbol{M}^{-1}\right)_{l_{1} l} \tilde{C}_{l} \sum_{l^{\prime}}\left(\boldsymbol{M}^{-1}\right)_{l_{2} l^{\prime}} \tilde{C}_{l^{\prime}}\right\rangle, \\
& =\sum_{l}\left(\boldsymbol{M}^{-1}\right)_{l_{1} l} \sum_{l^{\prime}}\left(\boldsymbol{M}^{-1}\right)_{l_{2} l^{\prime}}\left\langle\tilde{C}_{l} \tilde{C}_{l^{\prime}}\right\rangle .
\end{aligned}
$$

Using Eqs. (A.4) and (A.6), we find

$$
\begin{aligned}
\left\langle\tilde{C}_{l} \tilde{C}_{l^{\prime}}\right\rangle= & A+\frac{1}{(2 l+1)\left(2 l^{\prime}+1\right)} \\
& \times \sum_{m m^{\prime}}\left\langle\tilde{N}_{l m}^{j}\left(\tilde{N}_{l^{\prime} m^{\prime}}^{j}\right)^{*}\right\rangle\left\langle\tilde{N}_{l m}^{k}\left(\tilde{N}_{l^{\prime} m^{\prime}}^{k}\right)^{*}\right\rangle
\end{aligned}
$$

where $A$ denotes terms irrelevant to noise. Therefore, we need to estimate noise covariance $\left\langle\tilde{N}_{l m}^{j}\left(\tilde{N}_{l^{\prime} m^{\prime}}^{j}\right)^{*}\right\rangle$ in order to estimate Eq. (A.10). First, let us consider diagonal elements of noise covariance:

$$
\begin{aligned}
\left\langle\tilde{N}_{l m} \tilde{N}_{l m}^{*}\right\rangle= & \frac{16 \pi^{2}}{n_{\mathrm{pix}}^{2}} \sum_{i}\left\langle N_{i}^{2}\right\rangle\left|Y_{l m}^{i}\right|^{2} \\
& +\frac{32 \pi^{2}}{n_{\mathrm{pix}}^{2}} \sum_{i} \sum_{i^{\prime}>i}\left\langle N_{i} N_{i^{\prime}}\right\rangle Y_{l m}^{i}\left(Y_{l m}^{i^{\prime}}\right)^{*}
\end{aligned}
$$


The main contribution of Eq. (A.11) comes from the first term, because of cancellation through summation in the second term. Therefore, we find with good approximation:

$\left\langle\tilde{N}_{l m} \tilde{N}_{l m}^{*}\right\rangle \approx \frac{16 \pi^{2}}{n_{\mathrm{pix}}^{2}} \sum_{i}\left\langle N_{i}^{2}\right\rangle\left|Y_{l m}^{i}\right|^{2}$.

Off-diagonal elements of noise covariance are given by:

$$
\begin{aligned}
\left\langle\tilde{N}_{l m} \tilde{N}_{l^{\prime} m^{\prime}}^{*}\right\rangle= & \frac{16 \pi^{2}}{n_{\mathrm{pix}}^{2}} \sum_{i}\left\langle N_{i}^{2}\right\rangle Y_{l m}^{i}\left(Y_{l^{\prime} m^{\prime}}^{i}\right)^{*} \\
& +\frac{32 \pi^{2}}{n_{\mathrm{pix}}^{2}} \sum_{i} \sum_{i^{\prime}>i}\left\langle N_{i} N_{i^{\prime}}\right\rangle Y_{l m}^{i}\left(Y_{l^{\prime} m^{\prime}}^{i^{\prime}}\right)^{*} .
\end{aligned}
$$

Comparing (A.12) with Eq. (A.11), we may see the magnitude of off-diagonal elements is much smaller than that of diagonal elements, because cancellation through summation arise both in the first and the second term of Eq. (A.12). Therefore, we find noise covariance as follow:

$\left\langle\tilde{N}_{l m} \tilde{N}_{l^{\prime} m^{\prime}}^{*}\right\rangle \approx \delta_{l l^{\prime}} \delta_{m m^{\prime}} \frac{16 \pi^{2}}{n_{\text {pix }}^{2}} \sum_{i}\left\langle N_{i}^{2}\right\rangle\left|Y_{l m}^{i}\right|^{2}$.

Using Eqs. (A.8)-(A.10) and (A.13), we find covariance of $\hat{C}_{l}$ :

$$
\begin{aligned}
\operatorname{Cov}\left(\hat{C}_{l_{1}}, \hat{C}_{l_{2}}\right) \approx & C+\sum_{l}\left(\boldsymbol{M}^{-1}\right)_{l_{1} l}\left(\boldsymbol{M}^{-1}\right)_{l_{2} l} \times \frac{256 \pi^{4}}{n_{\mathrm{pix}}^{4}(2 l+1)^{2}} \\
& \times \sum_{m} \sum_{i i^{\prime}}\left\langle\left(N_{i}^{j}\right)^{2}\right\rangle\left\langle\left(N_{i^{\prime}}^{k}\right)^{2}\right\rangle\left|Y_{l m}^{i}\right|^{2}\left|Y_{l m}^{i^{\prime}}\right|^{2},
\end{aligned}
$$

where $C$ denotes terms irrelevant to noise. As shown in Eq. (A.14), we may neglect interpixel noise correlation in computing covariance of $\hat{C}_{l}$.

\section{References}

Ade, P., Bock, J., Bowden, M., et al. 2008, ApJ, 674, 22

Arfken, G. B., \& Weber, H. J. 2000, Mathematical Methods for Physicists, 5th Ed. (San Diego, CA USA: Academic Press)

Bond, J. R., Jaffe, A. H., \& Knox, L. 1998, Phys. Rev. D, 57, 2117
Brown, M. L., Ade, P., Bock, J., et al. 2009, ApJ, 705, 978

Bunn, E. F., Zaldarriaga, M., Tegmark, M., \& de Oliveira-Costa, A. 2003, 67, 023501

Das, S., Hajian, A., \& Spergel, D. N. 2009, Phys. Rev. D, 79, 083008

Dodelson, S. 2003, Modern Cosmology, 2nd Ed. (Academic Press)

Efstathiou, G. 2006, MNRAS, 370, 343

Eriksen, H. K., O’Dwyer, I. J., Jewell, J. B., et al. 2004, ApJ, 155, 227

Gorski, K. M., Hivon, E., Banday, A. J., et al. 2005, ApJ, 622, 759

Gorski, K. M., Wandelt, B. D., Hansen, F. K., Hivon, E., \& Banday, A. J. 1999 [arXiv: astro-ph/9905275]

Grain, J., Tristram, M., \& Stompor, R. 2009, Phys. Rev. D, 79, 123515

Halverson, N. W., Leitch, E. M., Pryke, C., et al. 2002, ApJ, 568, 38

Hinderks, J. R., Ade, P., Bock, J., et al. 2009, ApJ, 692, 1221

Hinshaw, G., Nolta, M. R., Bennett, C. L., et al. 2007, ApJ, 170, 288

Hivon, E., Górski, K. M., Netterfield, C. B., et al. 2002a, ApJ, 567, 2

Hivon, E., Górski, K. M., Netterfield, C. B., et al. 2002b, ApJ, 567, 2

Kamionkowski, M., Kosowsky, A., \& Stebbins, A. 1997, Phys. Rev. D, 55, 7368

Kim, J. 2007a, MNRAS, 375, 625

Kim, J. 2007b, MNRAS, 375, 615

Komatsu, E., Smith, K. M., Dunkley, J., et al. 2010, [arXiv: 1001.4538]

Kovac, J., Leitch, E. M., Pryke, C., et al. 2002, Nature, 420, 772

Kraus, J. 1986, Radio Astronomy, 2nd Ed. (Powell, Ohio USA: Cygnus-Quasar Books)

Larson, D., Dunkley, J., Hinshaw, G., et al. 2010, ApJS, accepted [arXiv: 1001.4635$]$

Leitch, E. M., Kovac, J. M., Pryke, C., et al. 2002, Nature, 420, 763

Leitch, E. M., Kovac, J. M., Halverson, N. W., et al. 2005, ApJ, 624, 10

Lewis, A. 2003, Phys. Rev. D, 68, 083509

Lewis, A., Challinor, A., \& Turok, N. 2002, Phys. Rev. D, 65, 023505

Liddle, A. R., \& Lyth, D. H. 2000, Cosmological Inflation and Large-Scale Structure, 1st Ed. (Cambridge University Press)

Mukhanov, V. 2005, Physical Foundations of Cosmology, 1st Ed. (Cambridge University Press)

Ng, K., \& Liu, G. 1999, Int. J. Mod. Phys. D, 8, 61

Nolta, M. R., Dunkley, J., Hill, R. S., et al. 2009, ApJS, 180, 296

Pryke, C., Halverson, N. W., Kovac, J. M., et al. 2002a, ApJ, 568, 28

Pryke, C., Halverson, N. W., Leitch, E. M., et al. 2002b, ApJ, 568, 46

Pryke, C., Ade, P., Bock, J., et al. 2009, ApJ, 692, 1247

Riley, K. F., Hobson, M. P., \& Bence, S. J. 2006, Mathematical Methods for Physics and Engineering: A Comprehensive Guide, 3rd Ed. (Cambridge University Press)

Rohlfs, K., \& Wilson, T. L. 2003, Tools of Radio Astronomy, 4th Ed. (New York, NY USA: Springer-Verlag)

Seljak, U., \& Hirata, C. M. 2004, Phys. Rev. D, 69, 043005

Smith, K. M. 2006, Phys. Rev. D, 74, 083002

The Planck Collaboration 2006, [arXiv: astro-ph/0604069]

Tucci, M., Martínez-González, E., Vielva, P., \& Delabrouille, J. 2005, MNRAS, 360,935

Wandelt, B. D., Hivon, E., \& Górski, K. M. 2001, Phys. Rev. D, 64, 083003

Zaldarriaga, M. 1998, ApJ, 503, 1

Zaldarriaga, M., \& Seljak, U. 1997, Phys. Rev. D, 55, 1830 